\section{REDIMAT}

Journal of Research in Mathematics Education
Hipatia Press

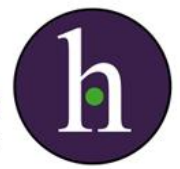

Instructions for authors, subscriptions and further details:

http://redimat.hipatiapress.com

\title{
El Problema Didáctico del Álgebra Elemental: Un Análisis Macro-Ecológico desde la Teoría Antropológica de lo Didáctico
}

Noemí Ruiz Munzón ${ }^{1}$, Marianna Bosch², and Josep Gascón ${ }^{3}$

1) Universitat Pompeu Fabra, Spain.

2) Universitat Ramon Llull, Spain.

3) Universitat Autònoma de Barcelona.

Date of publication: June $24^{\text {th }}, 2015$

Edition period: June 2015-October 2015

To cite this article: Ruiz Munzón, N., Bosch, M., \& Gascón, J. (2015). El problema didáctico del algebra elemental: Un análisis macro-ecológico desde la teoría antropológica de lo didáctico. REDIMAT, Vol 4(2), 106-131. doi: 10.17583/redimat.2015.1386

To link this article: http://dx.doi.org/10.17583/redimat.2015.1386

\section{PLEASE SCROLL DOWN FOR ARTICLE}

The terms and conditions of use are related to the Open Journal System and to Creative Commons Attribution License (CC-BY). 


\section{The Didactical Problem of Elementary Algebra: A Macro- Ecological Analysis Based on the Anthropological Theory of Didactics}

Noemí Ruiz Munzón

Universitat Pompeu Fabra

Josep Gascón

Universitat Autònoma de Barcelona
Marianna Bosch

Universitat Ramon Llull

(Received: 16 January 2015; Accepted: 10 June 2015; Published: 24 June 2015)

\section{Abstract}

We present a global, synthetic and updated vision of the contributions made by the anthropological theory of the didactic to the problem of teaching elementary algebra. We start by summarising the first results obtained by Yves Chevallard in the 80s that have marked the trend of research on elementary algebra in the anthropological approach. A reference epistemological model of elementary algebra is then proposed. This model provides a new interpretation of the role of elementary algebra in current school mathematics and guides the study of its ecology, that is, the constraints which partially explain the current situation and the conditions that could modify it in a given direction.

Keywords: Anthropological theory of the didactic, elementary algebra, didactic ecology, stages of algebraization, secondary school 


\section{El problema Didáctico del Álgebra Elemental: Un Análisis Macro-Ecológico desde la Teoría Antropológica de lo Didáctico}

Noemí Ruiz Munzón

Universitat Pompeu Fabra

Josep Gascón

Universitat Autònoma de Barcelona
Marianna Bosch

Universitat Ramon Llull

(Recibido: 16 Enero 2015; Aceptado: 10 Junio 2015; Publicado: 24 Junio 2015)

\section{Abstract}

Presentamos una visión global, sintética y actualizada de las aportaciones realizadas por la teoría antropológica de lo didáctico al problema de la enseñanza del álgebra elemental. Partimos de los primeros resultados obtenidos entre 1980 y 1990 por Yves Chevallard que marcaron la línea de investigación en torno al álgebra elemental en el enfoque antropológico. Construimos un modelo epistemológico de referencia del álgebra elemental que permite reinterpretar su papel en la matemática escolar actual y estudiar su ecología, esto es, las restricciones que explican en parte dicha situación y las condiciones que permitirían modificarla en una dirección determinada.

Keywords: Teoría antropológica de lo didáctico, algebra elemental, ecología didáctica, etapas de algebrización, enseñanza secundaria 


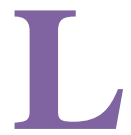

a mayoría de investigaciones didácticas sobre el álgebra elemental se centran en estudiar las principales dificultades de los alumnos al inicio del aprendizaje y las posibles actuaciones del profesor (o de la enseñanza) para paliarlas. Así, por ejemplo, los trabajos de Booth (1984), Kieran (1981), Vergnaud (1985) estudian las creencias iniciales de los alumnos en torno al signo de igualdad y las dificultades que éstas comportan para el aprendizaje de las ecuaciones. Los trabajos de Bednarz (2001), Filloy y Rojano (1989), entre otros, analizan las dificultades con las que se encuentran los alumnos al operar con incógnitas debido a la falta de comprensión sobre la invariancia del conjunto de soluciones de una ecuación si se aplican transformaciones equivalentes a ambos lados de ésta. Y en el ámbito de la teoría de modelos locales (Filloy, Rojano y Puig, 2008) se analizan, de manera específica, distintos componentes de los fenómenos de la matemática educativa: los de competencia formal, de procesos cognitivos y de comunicación.

Es mucho más difícil encontrar, en la investigación educativa, trabajos que examinen qué es lo que se enseña bajo el apelativo de álgebra elemental y, en consecuencia, qué se entiende por "álgebra elemental" en la clase de matemáticas, en la escuela y, más ampliamente, en la sociedad. Esta pregunta inicial - qué actividades y conocimientos constituyen el álgebra elemental en cuanto saber enseñado - y su versión complementaria - qué actividades y conocimientos relativos al álgebra no se enseñan en la escuela - constituyen sin embargo un cuestionamiento esencial para indagar las condiciones de posibilidad de un cambio educativo que no se reduzca a una mera innovación local (Chevallard y Bosch, 2012). Este cuestionamiento constituye lo que denominamos la ecología didáctica del sistema de enseñanza relativo al álgebra elemental (Artigue et al, 2001). Se sitúa en el corazón del desarrollo de la teoría antropológica de lo didáctico (en adelante TAD), desde los primeros trabajos sobre la transposición didáctica (Chevallard, 1985/1992) y aquellos más específicos sobre la transposición didáctica del álgebra elemental (Chevallard, 1984, 1989, 1990). Estos trabajos dieron lugar a la construcción de un ámbito de investigación didáctica en el que se inscriben numerosas investigaciones, como Gascón (1993, 1994-95, 1999); Bolea, Bosch \& Gascón (2001a, 2001b); y las recogidas en el volumen especial de la revista Recherche en Didactique des Mathématiques (Coulange et al 2012); Assude et al (2012); 
Chevallard y Bosch (2012); Grugeon et al (2012); Ruiz-Munzón et al (2012).

En este trabajo trataremos tres problemas fuertemente interrelacionados que forman parte de las diferentes dimensiones del problema didáctico del álgebra elemental (Gascón, 2011). En primer término indagaremos qué se entiende por álgebra en el sistema educativo, para ello en la sección 2 propondremos una panorámica simplificada de la evolución que ha sufrido el álgebra elemental como saber a enseñar en el sistema de educación español desde finales del siglo XIX hasta la actualidad, lo que nos permitirá empezar a delimitar las "restricciones transpositivas" vía el estudio de los programas y currículos oficiales que explic an parcialmente el estado actual del álgebra elemental como saber a enseñar. En la sección 3 propondremos un modelo epistemológico de referencia (MER) para el álgebra elemental como alternativa al modelo implícito vigente actualmente en la enseñanza secundaria. Utilizando el MER como sistema de referencia, analizaremos algunos fenómenos didácticos asociados al citado modelo epistemológico implícito en la enseñanza secundaria. Por fin, en la sección 4, analizaremos algunas de las principales condiciones que permiten y las restricciones que inciden sobre la génesis, desarrollo y difusión del álgebra elemental y que deberán tenerse presentes si pretendemos cambiar, en una dirección determinada, la actual situación de dicho ámbito de la actividad matemática. Para ello utilizaremos la escala de niveles de codeterminación didáctica (Chevallard, 2002) que permite situar las diferentes condiciones y restricciones en el nivel en el que surgen, clarificando así el grado de responsabilidad de las diversas instituciones involucradas.

\section{Evolución de la Enseñanza del Álgebra Elemental en España}

Siguiendo el guión de análisis que se presenta en Chevallard (1984), estudiaremos de manera muy esquemática la evolución curricular del álgebra elemental en España para identific ar el origen, el estatus y la razón de ser de esta área de las matemáticas escolares que, como veremos, ha sufrido fuertes fluctuaciones a lo largo del tiempo y mantiene una posición incierta en el currículum actual. 


\section{El Álgebra Elemental en la Enseñanza Tradicional de las Matemáticas}

Antes de la reforma de las matemáticas modernas, en los años 60, el álgebra representaba, en el currículum escolar, la entrada a las "matemáticas avanzadas". Las matemáticas de la enseñanza primaria -0 "primera enseñanza" como se designó a partir de la Ley Moyano de 1857se limitaban al corpus tradicional de la aritmética práctica, con sus cuatro reglas y el sistema legal de medidas, las fracciones o quebrados y el universo de las razones y proporciones junto con la regla de tres. Se le añadían en la primaria superior, principios de geometría, dibujo lineal y agrimensura. La enseñanza del álgebra no llegaba hasta el segundo curso de secundaria (13-14 años), donde la matemática se dividía en los tres bloques tradicionales de aritmética, álgebra y geometría. Esta es la organización clásica de las matemáticas elementales, que encontramos en la mayoría de sistemas educativos occidentales anteriores a la reforma de las Matemáticas Modernas, estructura que en España pervivió durante más de 100 años, hasta la aparición de la Ley General de Educación de 1970.

En esta organización clásica de las matemáticas enseñadas, el álgebra mantiene habitualmente una estructura estándar que incluye una introducción al cálculo algebraico y a los números negativos (también llamados "algebraicos"), el tratamiento de las ecuaciones de primer grado y los problemas asociados, el cálculo de potencias y raíces de expresiones algebraicas, el tratamiento de las ecuaciones de segundo grado y los problemas asociados, entre otros.

\section{La Reforma de las Matemáticas Modernas}

Con la llegada de la reforma de las matemáticas modernas a partir de los años 60, la organización tradicional de las matemáticas sufre un fuerte descalabro. De la "ciencia de la cantidad" organizada en tres bloques, se pasa a una nueva concepción del edificio matemático como conjunto de estructuras y se reconstruye la matemática enseñada a partir de principios lógicos como indica González (2006). De este modo, en los nuevos programas del bachillerato (o enseñanza secundaria) publicados en 1967 ya no se encuentra ningún rastro de los tres bloques de contenido tradicionales y sólo se indica una serie lineal de lecciones o temas sin ninguna agrupación aparente. 
En esta nueva organización de los contenidos no se mencionan las expresiones algebraicas que, a diferencia de los polinomios, no se saben inscribir en ninguna estructura conjuntista concreta. La noción de aplicación entre conjuntos y de función numérica ocupa una posición central, a la que queda supeditado el cálculo ecuacional. Permanecen sin embargo algunos vestigios de la antigua organización matemática como la noción de proporcionalidad (de magnitudes y de segmentos) que coexistirá durante años con la de aplicación lineal.

\section{La Estructura Curricular de la Matemática 'Postmoderna' en España}

Unos años más tarde, en la propuesta curricular que fija las enseñanzas mínimas para el ciclo superior de la Educación General Básica (alumnos entre 12 y 14 años), la matemática enseñada adquiere una nueva estructuración en ocho bloques de contenidos: Conjuntos numéricos, Divisibilidad en $\mathbb{N}$, Geometría plana, Funciones, Proporcionalidad de magnitudes, Geometría del espacio, Estadística descriptiva e Informática (Real Decreto 3087/1982). En esa nueva división, los temas que tradicionalmente correspondían al álgebra quedan esparcidos por los distintos bloques. Los números enteros pasan a formar parte del bloque de conjuntos numéricos y el trabajo con expresiones algebraicas y ecuaciones se incluye en el apartado de las funciones. Los programas que se desarrollan con base en la Ley Orgánica General del Sistema Educativo (LOGSE) de 1990, consolidan la ruptura con la estruc tura tripartita clásica de la aritmética-álgebra-geometría y es el universo de las funciones el que adquiere, en cierto sentido, el papel de entrada a la "matemática superior" que anteriormente correspondía al álgebra.

\section{El Álgebra Elemental en los Currículos Actuales}

En España, la reforma curricular de la Ley Orgánica de Educación (2/2006) propone una nueva distribución de los contenidos que vuelve a introducir el álgebra como uno de los bloques curriculares junto con "Números", "Geometría", "Funciones y gráficas", "Estadística y probabilidad" y "Resolución de problemas". Esta estructura no resulta ser demasiado estable. Por ejemplo, el currículo de Catalunya adoptará un recorte en cinco 
bloques: "Numeración y cálculo", "Relación y cambio", "Espacio y forma", "Medida", "Estadística y azar."

\section{Características del Álgebra como Saber Enseñado}

Al examinar los libros de texto más utilizados en la enseñanza secundaria española actual, vemos que los contenidos relacionados con el álgebra se centran principalmente en el desarrollo de cálculo ecuacional, con una "introducción al lenguaje algebraico" y una culminación en la resolución de problemas verbales. Aunque el desarrollo del cálculo algebraico está ligado históricamente a la teoría de ecuaciones, la enseñanza actual presenta una desproporción sorprendente entre los ejercicios de cálculo algebraico propuestos a los alumnos y los cálculos algebraicos efectivamente exigidos en la resolución de ecuaciones: éstos son en general mucho más simples que aquéllos. El tema de las ecuaciones no basta pues para justificar la importancia dada al cálculo algebraico en la ESO.

Un primer beneficio de las inversiones realizadas en el cálculo algebraico de Secundaria, se recoge con el cálculo de límites y de derivadas que aparecen por primera vez en el Bachillerato. Pero, a falta de otro ámbito de intervención adaptado, el cálculo algebraico se hace siempre en el vacío, sin relación con un objetivo matemático que convertiría el cálculo en un medio (y no un fin en sí mismo) y que daría pertinencia a las manipulaciones efectuadas sobre las expresiones algebraicas. En resumen, el primer aprendizaje del cálculo algebraico se hace en un marco formal y no funcional, en el que el alumno aprende a "desarrollar", "factorizar", "simplificar" expresiones como objetivo en sí mismo, no como medio para resolver o facilitar alguna tarea.

En síntesis, podemos concluir que la enseñanza actual mantiene las dos limitaciones que indica Chevallard (1984) sobre el aprendizaje "formal" de las técnicas algebraicas:

1. Se aprende implícitamente en referencia a la aritmética y al cálculo con números, cuando en realidad el cálculo algebraico se rige por unas reglas sintácticas muy diferentes a las del cálculo aritmético. En particular, el cálculo aritmético tiende a simplificar cualquier operación realizada antes de pasar al cálculo siguiente. En cambio el álgebra se basa en la no 
reducción - e incluso en la "complejización" - ostensiva de los cálculos y manipulaciones realizadas (Bosch, 1994).

2. El aprendizaje formal es incapaz de recrear toda la variedad de manipulaciones que el alumno puede necesitar en el momento en que deba hacer un uso funcional de la herramienta algebraica. Surge así todo un vocabulario ligado a la necesidad de disponer de consignas que se refieren a las distintas manipulaciones formales ("calcular", "simplificar", "desarrollar", "factorizar" y todas sus variantes) que, no sólo no permiten generar las manipulaciones algebraicas fundamentales, sino que, además, impiden que el alumno se enfrente al problema de tener que elegir cuál es, en cada caso, la transformación formal más adecuada para alcanzar al fin propuesto.

\section{Un Modelo Epistemológico de Referencia del Álgebra Elemental}

En este apartado elaboraremos un modelo epistemológico de referencia (en adelante, MER) utilizando los datos empíricos que emanan tanto del desarrollo histórico del álgebra en la matemática sabia como de su evolución como saber por enseñar. La construcción de un MER del álgebra elemental tiene, al menos, dos objetivos: en primer lugar será útil para reinterpretar lo que se considera "álgebra" en la matemática escolar actual (en el ámbito de la enseñanza secundaria) y para indagar el papel que desempeña el álgebra elemental en relación con las demás áreas; y, en segundo lugar, el MER será imprescindible para estudiar la ecología institucional de este ámbito de la matemática escolar.

\section{Evolución del Saber Sabio}

Siguiendo a Chevallard (1984), partimos de considerar como "núcleo primario" del álgebra la teoría de ecuaciones tal como la crea al-Khwarizmi (c. 780 - c. 850) en la primera parte del siglo IX. Desde el punto de vista que adoptamos aquí, la evolución histórica del álgebra sabia se puede esquematizar en tres grandes etapas, dejando de lado los problemas del simbolismo algebraico que se zanjan esencialmente entre finales del XVI y 
principios del XVII, aunque no se resolverán completamente hasta el siglo XIX con la construcción formal de los sistemas numéricos.

La primera etapa corresponde a la identificación del álgebra, como sector de las matemáticas sabias, con la resolución de "problemas aritméticos" (expresados tradicionalmente de forma retórica). Esta identificación no se extiende más allá del Renacimiento porque estos problemas, exc eptuando los del corpus diofántic o que seguirán alimentando la reflexión matemática, se resuelven teóricamente quedando reducidos a simples aplicaciones comerciales. Es lo que da lugar a la aritmética práctica o comercial, del que la Arithmetica Universalis de Newton (1707) es un ejemplar ilustre y que, como hemos visto, constituye el corazón del corpus tradicional de la aritmética enseñada clásica y pervivirá en nuestros sistemas de enseñanza hasta la reforma de las matemáticas modernas.

La segunda etapa corresponde al desarrollo de la teoría de ecuaciones que ocupará a los algebristas europeos desde el Renacimiento hasta Descartes y su geometría (1673/1925). Con la logística especiosa de Viète (1540-1603) el álgebra aparecerá ya como un método completo para operar sobre las especies o las formas de las cosas, y la aritmética, la logística numerosa, como una técnica que se ocupa de los números. El álgebra se transforma así en el estudio de los tipos generales de formas y de ecuaciones, tomando como objeto de estudio el propio cálculo algebraico y constituyéndose como una nueva área de las matemáticas. Los algebristas italianos del siglo XVI (Scipione del Ferro, Tartaglia, Cardano, Bombelli, etc.) resolvieron las ecuaciones generales de grados 3 y 4; sus sucesores chocarán durante tiempo con las ecuaciones de grado superior hasta que, entre finales del XVIII (Vandermonde, Lagrange) y principios del XIX (Abel, Galois), se llega a una respuesta negativa (imposibilidad de resolver algebraicamente, "por radicales", las ecuaciones de grado superior o igual a 5). De este trabajo surgirán, como es bien sabido, los primeros conceptos del álgebra moderna (cuerpos, anillos, etc.) que inician un nuevo periodo de desarrollo de la matemática sabia.

En la tercera etapa que distinguimos aquí, la herramienta que constituye el cálculo algebraico bajo su forma simbólica moderna penetra todo los ámbitos de la matemática y encuentra nuevos campos de aplicación, primero en la geometría (a partir de Descartes) y la teoría de números (con Fermat) y después en el análisis matemático (que inicialmente se designaba como "análisis algebraico") con la creación, a finales del siglo XVII, del 
cálculo infinitesimal. En este periodo moderno que llega hasta hoy día, podemos decir, siguiendo a Bolea (2003), que todos los ámbitos de la matemática sabia actual están completamente algebrizados en el sentido de que es difícil encontrar actividades que se desarrollen sin recurrir al simbolismo algebraico. Y es también esta matemática completamente algebrizada la que los alumnos acabarán por frecuentar, de forma abrupta, al final de la educación secundaria.

\section{Construcción de un Modelo Epistemológico de Referencia: El Álgebra Elemental como Instrumento de Modelización}

En clara afinidad con la evolución histórica y, siguiendo a Bolea, Bosch y Gascón (1998), podemos interpretar en primera instancia el álgebra elemental como una herramienta que permite llevar a cabo una actividad de modelización que acaba por afectar a todos los sectores de la matemática mediante un proceso que denominamos proceso de algebrización.

Esta visión del álgebra como proceso de algebrización permite dar una respuesta concreta al problema del estatus y la razón de ser del álgebra elemental en la enseñanza secundaria actual. Por un lado, el álgebra aparece como una herramienta privilegiada para abordar cuestiones teóricas que surgen en distintas áreas de la matemática elemental (especialmente la aritmética y la geometría) y que no encuentran respuesta dentro de estas mismas áreas. Un ejemplo tipo y bien conocido es el de trabajar con patrones o secuencias de las que se conoce el principio de construc ción pero no la fórmula o ley general que las define. Esta caracterización pone de manifiesto otro rasgo diferenciador del álgebra elemental en relación con la aritmética: el álgebra elemental como "aritmética universal" permite estudiar relaciones universales independientemente de la naturaleza de los objetos relacionados. Como consecuencia se obtienen resoluciones "generalizadas", de todo un tipo de problemas, y no únicamente la respuesta asociada a un problema aislado como ocurre en aritmética. Por tanto, otro aspecto esencial de la razón de ser del álgebra elemental es la de responder a la necesidad de agrupar las tareas en tipos de problemas e introducir la idea de generalización del proceso de resolución. Esta interpretación del álgebra elemental permite integrar la mayor parte de puntos de vista con los que se presenta el álgebra en el currículo de 
secundaria (Puig, 2012) y, en particular, el que propone una entrada al álgebra a través de la generalización (Sesa, 2005).

En Ruiz-Munzón (2010) se propone una concreción de este proceso de algebrización articulado en tres etapas. El punto de partida del proceso requiere disponer de un sistema inicial para modelizar. Este sistema agrupa lo que Chevallard designó, a principios de los años 2000, como los programas de cálculo (PC) es decir cualquier secuencia de operaciones aritméticas que se puede efectuar "paso a paso". Podemos considerar que la mayoría de problemas de la aritmética (y algunos de la geometría elemental) remiten a la descripción verbal (o gráfica) y efectuación de estos programas de cálculo. El estudio de estos sistemas de PC hace surgir cuestiones de naturaleza teórica relacionadas con la necesidad de explicar las razones por las cuales se obtiene un resultado dado, de justificar o interpretar resultados equivalentes, etc. Es esta necesaria y progresiva ampliación de la problemática en torno a los PC la que tomaremos como base para describir las tres etapas sucesivas del proceso de algebrización que constituyen una formulación esquemática del MER del álgebra elemental que proponemos.

La primera etapa ${ }^{1}$ del proceso de algebrización aparece cuando es necesario considerar el programa de cálculo no sólo como un proceso sino como un todo, traduciendo la formulación retórica del PC a una formulación escrita. Esto no implica, necesariamente, el uso de letras para denotar los números o las cantidades involucradas en el programa (denominadas como variables o argumentos del PC), pero sí requiere poner en juego la jerarquía de las operaciones y las normas de uso de paréntesis. En el trabajo en esta etapa aparecen nuevas técnicas para la creación y la simplificación de escrituras y un nuevo entorno tecnológico-teórico que incluye la noción de expresión algebraica y de PC equivalentes. Siguiendo la terminología clásica del cálculo ecuacional, diríamos que en esta etapa se incluye la técnica que al-Khwarizmi designó por al-muqabala, pero no la operación fundamental de "restauración". "cancelación" o $a l$-jabr, palabra árabe que da nombre al álgebra y que consiste en transformar simultáneamente los dos PC (los dos miembros de la ecuación) para obtener una nueva ecuación (o igualdad entre PC) equivalente a la anterior.

El paso a la segunda etapa del proceso de algebrización se produce cuando las técnicas de simplificación de un PC y el trabajo con PC equivalentes no es suficiente para resolver el problema propuesto porque 
los "datos" del problemas y la propia "incógnita" vienen dados en forma de relaciones entre variables del PC. En consecuencia, la estructura de los tipos de problemas característicos de esta segunda etapa viene dada mediante la igualdad entre dos $\mathrm{PC}$ con los dos mismos argumentos no numéricos $\left(x_{1}, x_{2}\right)$, por ejemplo igualando: $\mathrm{PC}_{1}\left(2, x_{1}, x_{2}\right)=2 x_{1}+x_{2} \mathrm{y}$ $\mathrm{PC}_{2}\left(5,7, x_{1}, x_{2}\right)=7 x_{1}-5 x_{2}$. En esta etapa aparece una mayor complejidad en las técnicas algebraicas de la primera etapa y, además, se requieren nuevas técnicas que permitan la manipulación de expresiones algebraicas, en particular, el cálculo ecuacional y la consideración de las ecuaciones (en principio con dos variables) como un nuevo objeto matemático. En el caso particular de que la incógnita sea el valor numérico que toma uno de los argumentos no numéricos (dados el valor concreto que toma el otro argumento no numérico y la relación entre los dos PC), entonces el problema se reduce a la resolución de una ecuación con una incógnita. Es precisamente en este ámbito muy particular de la segunda etapa donde se sitúa principalmente el álgebra de la escuela secundaria española, esto es, en la resolución de ecuaciones de primer y segundo grado con una incógnita y de situaciones que pueden ser modelizadas por éstas.

La tercera etapa de algebrización llega cuando no se limita el número de variables con las que trabajamos y se elimina la distinción entre parámetros e incógnitas. Los PC que intervienen en esta tercera etapa como, por ejemplo, $\mathrm{PC}(2,3, \pi, r, R, h, H)=\pi\left(R^{2} H-r^{2} h\right) / 3$, pueden ser interpretados como "fórmulas" o modelos algebraicos de un sistema matemático o extramatemático. El trabajo con dichos modelos persigue aumentar los conocimientos sobre el sistema modelizado mediante el estudio de cuestiones problemátic as que no pueden disponerse en el ámbito del sistema.

Aunque debemos precisar que las técnicas algebraicas accesibles actualmente en secundaria para abordar las cuestiones de estas dos últimas etapas son muy pobres y poco eficaces una vez nos alejamos del mundo lineal y cuadrático, hay que recordar que antiguamente dicha técnicas se integraban en lo que se denominó "álgebra superior" y jugaron un papel importante en la transición del álgebra elemental al cálculo diferencial.

Utilizando este MER como sistema de referencia podemos afirmar que el álgebra escolar tal como se presenta en la ESO española se ubica completamente en la parte más elemental de la segunda etapa del proceso de algebrización y que nunca se alcanza la tercera etapa del mismo, puesto 
que el álgebra escolar no es utilizada habitualmente como una herramienta de modelización para el estudio de problemas de todo tipo. En efecto, en la matemática escolar de la enseñanza secundaria, el objetivo final, la razón de ser, del estudio del álgebra escolar consiste en traducir el enunciado de un problema al lenguaje algebraico y resolverlo mediante el planteamiento de una ecuación con una incógnita. Como fue constatado por Bolea (2003) la ausencia de una manipulación sistemática de la estructura global de los problemas de Secundaria se ve reflejada en el hecho de que las "letras" que forman parte de una expresión algebraica juegan únicamente el papel de incógnitas (en las ecuaciones) o únicamente el papel de variables (en el lenguaje funcional), pero los parámetros están prácticamente ausentes.

De acuerdo con el MER que hemos propuesto como sistema de referencia, consideramos que, si se pretende que en la enseñanza secundaria (e incluso en la primaria) sea posible llevar a cabo una actividad sistemática y progresiva de modelización algebraica, entonces sería útil organizar un proceso de estudio que cubriese sucesivamente las tres etapas del proceso de algebrización descritas en el MER.

\section{El Problema de la Ecología del Álgebra Elemental}

Después de proponer un MER que permite reinterpretar cómo es el álgebra que se propone para ser enseñada en la Secundaria española actual, conviene intentar explicar, al menos parcialmente, por qué el álgebra elemental está en la situación descrita y cuáles son las restricciones que inciden sobre ella. Dicho en otros términos, después de dar una respuesta provisional a la dimensión epistemológica del problema del álgebra elemental mediante la construcción de un MER (\$3) y una vez hemos respondido a algunas de las cuestiones que forman parte de la dimensión económica, esto es, cuestiones relativas a cuáles son las actividades y conocimientos que constituyen el álgebra elemental en cuanto saber enseñado, (\$2), hemos de formular adecuadamente y empezar a abordar la dimensión ecológica de dicho problema (Gascón, 2011).

Para analizar las condiciones ecológicas que permiten que determinados objetos y actividades puedan existir en la escuela, Chevallard (2007) introdujo la noción de escala de niveles de codeterminación didáctica que amplía y al mismo tiempo estructura el ámbito empírico que el investigador en didáctica debe tomar en consideración. La manera de organizar los 
contenidos matemáticos, que llamaremos más adelante organizaciones (o praxeologías) matemáticas, y los dispositivos y gestos que se necesitan para su enseñanza (las organizaciones didácticas) requiere que éstos cumplan una serie de condiciones que pueden ser específicas de la disciplina en cuestión (en nuestro caso las matemáticas) o bien genéricas, es decir, provenientes de la manera de organizar las actividades de enseñanza y

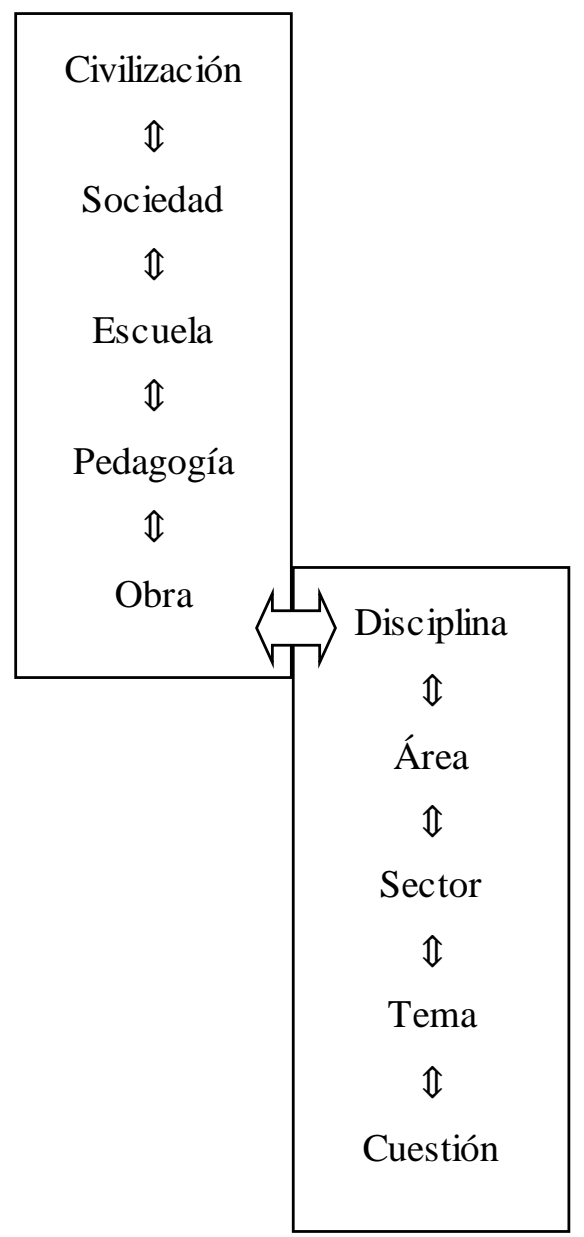

Figure 1. Niveles de codeterminación didáctica 
aprendizaje en la escuela, los roles que se asigna a la escuela en la sociedad, etc. Estas condiciones se estructuran de forma jerárquica según muestra el esquema de la Figura 2. Las condiciones que se imponen en los distintos niveles de codeterminación didáctica, a la vez que hacen posible el desarrollo de determinadas actividades, también restringen el universo de acciones posibles.

Los trabajos sobre la enseñanza del álgebra elemental realizados en la TAD han permitido identificar importantes restricciones provenientes de todos los niveles de la jerarquía de codeterminación, inc luyendo los niveles superiores, de la civilización y la sociedad (\$4.1) y los niveles más específicos de la disciplina (\$4.2).

\section{Restricciones Provenientes de los Niveles de la Civilización y Sociedad}

La teoría de la transposición didáctica parte de la constatación de que la sociedad tiende a exigir del sistema de enseñanza que el saber que es propuesto para ser enseñado al ciudadano sea compatible tanto con el saber sabio que lo legitima como con la epistemología cultural corriente. En nuestro caso, la cultura occidental se sustenta sobre la postura metafísica del logocentrismo, según el término acuñado por el filósofo francés Jacques Derrida (1967), que asume, más o menos explícitamente, que el pensamiento reside en la cabeza, que éste se expresa en voz alta por medio de la palabra y se conserva posteriormente mediante la escritura. Por lo tanto, la escritura es vista como una "degradación" del pensamiento o, a lo sumo, como un producto secundario del mismo: primero se piensa y se "concibe"; después se plasma el pensamiento en el papel (o la pantalla). Desde este punto de vista, no se valora suficientemente el papel que pueden desempeñar los formalismos científicos (productos primariamente escritos y que se "oralizan" posteriormente) como instrumentos de pensamiento científico. No se entiende la necesidad de recurrir a grafismos que no son abreviaciones de conceptos verbales, sino signos escritos productores de significados por sí mismos. El álgebra constituye, en cierto sentido, el primer producto de esta escritura matemática primaria. El cálculo algebraico y, en general, el trabajo con expresiones algebraicas, no es una obra fácilmente "culturizable" (en el sentido de asumible por la cultura 
corriente) ya que constituye un formalismo que ha nacido como lenguaje escrito y no tiene siempre un referente claro en el discurso verbal.

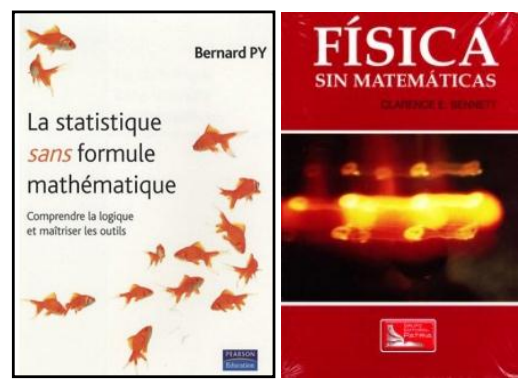

Figure 2. Obras de divulgación

No desarrollaremos más aquí esta fuente de incomprensión cultural hacia la importancia de la "instrumentalidad" del formalismo científico como herramienta de pensamiento, que encontramos bajo distintas expresiones, en diversos ámbitos sociales. Las obras de divulgación con títulos como "Física sin matemáticas" o incluso "La estadística sin fórmulas" (Figura 3) ya hablan por sí solas. Lo que queremos subrayar aquí es la importante incidencia de esta postura metafísica sobre los niveles inferiores de codeterminación didáctica, puesto que el alumno no podrá ver rec onocidos sus cálculos como "razonamientos" y el profesor deberá buscar nuevos "significados" a esta herramienta que aparece siempre como una pura "sintaxis" sin una "semántica" propia. Esta peyoración cultural del álgebra queda claramente reflejada en esta cita del eminente geómetra británico contemporáneo Michael Atiyah (2002, pp. 42-43, la cursiva es nuestra):

El Álgebra es la oferta que el diablo hace a los matemáticos. El diablo dice: Te daré esta poderosa máquina que responderá a cualquier pregunta que desees. Todo lo que necesitas hacer es darme tu alma, olvídate de la Geometría y te daré esta maravillos a máquina. Por supuesto nos gustaría tener ambas cosas [...]. No obstante el daño a nuestra alma está ahí, porque cuando entras en 
cálculos algebraicos esencialmente dejas de pensar geométricamente, dejas de pensar en el significado.

Es interesante apuntar que muchas de las investigaciones didácticas sobre las dificultades en el aprendizaje del álgebra elemental no escapan a este logocentrismo occidental. De ahí que, durante muchos años, una problemática frecuente de estas investigaciones se haya centrado en estudiar los "significados" que los alumnos atribuyen al simbolismo algebraico. Así, por ejemplo, encontramos un gran número de trabajos dedicados al estudio de las dificultades de los alumnos para "entender" - es decir, verbalizar - nociones como las de ecuación, igualdad, identidad, parámetro, etc. o, más ampliamente, investigaciones que tratan del estudio de las concepciones espontáneas de los alumnos respecto a conceptos fundamentales del álgebra, como por ejemplo: los signos “+”, “_“ y "=” (Behr, Erlwanger y Nichols, 1980; Kieran, 1981) o las letras utilizadas para designar las incógnitas, variables o parámetros (Trigueros et al., 1996; Blanco y Garrote, 2007; Malisani y Spagnolo, 2009). Por su parte, Sfard y Linchevski (1994), postulan que el origen de muchas de las dificultades de los alumnos radica en que tienden a adjudicar un significado rígido a una expresión algebraica, mientras que el profesor puede interpretarla desde puntos de vista muy diferentes en función de la situación o el problema de que se trate.

Esta concentración de las investigaciones relativas al álgebra elemental en la función semiótica del lenguaje algebraico y la consiguiente ignorancia casi absoluta de la función instrumental del mismo (Bosch, 1994) constituye un síntoma de la vigencia del logocentrismo e impide que, también en el ámbito de la investigación didáctica, se interprete el álgebra elemental como un instrumento de modelización tal como propone el MER que hemos propuesto como sistema de referencia.

\section{Restricciones Provenientes del Nivel de la Disciplina}

La vinculación del álgebra con lo numérico está condicionada por considerar que el estudio de la aritmética debe situarse en el currículo escolar antes que el estudio del álgebra. Uno de los argumentos sobre los que se sustenta esta decisión es la consideración de la aritmética como "más concreta" y, por lo tanto, más fácil que el álgebra, siendo ésta segunda más 
"abstracta" y difícil. Los defensores de esta cronología temporal argumentan que el estudio del álgebra requiere un pensamiento formal y que este tipo de pensamiento se desarrolla en etapas avanzadas de evolución del alumno (Lins y Kaput, 2004).

Chevallard (1989) muestra que, en coherencia con la evolución del saber sabio, la función principal del álgebra no es la de generalizar la aritmética sino la de modelizar sistemas intra-matemáticos o extra-matemáticos. Afirma que la enseñanza del álgebra debe promover una dialéctica entre el manejo formal del cálculo algebraico y el contenido de los sistemas numéricos. Este objetivo se deriva en una doble consideración: no podemos tener un dominio funcional del cálculo algebraico, sin ponerlo en funcionamiento como una herramienta útil; y no podemos poner en funcionamiento esta herramienta sin instaurar una verdadera dialéctica entre lo numérico y lo algebraico.

Puntualicemos que esta función modelizadora no niega la relación fundamental que existe entre el álgebra y la aritmética, pero sí la jerarquía unidirec cional preestablecida entre estos dos ámbitos matemátic os. Desde esta nueva interpretación, la aritmética, o al menos parte de ella, constituye un sistema intra-matemático, entre otros, que el instrumento algebraico puede modelizar.

Diversas investigaciones en el seno de la TAD (Gascón, 1993, 1994-95; Bolea, Bosch, y Gascón, 1998) han cuestionado explícitamente el modelo epistemológico-didáctico del álgebra dominante en las instituciones escolares. En su tesis, Bolea (2003), a partir de la revisión de documentos oficiales de la LOGSE y diversos libros de texto, caracteriza la interpretación habitual del álgebra escolar como una aritmética generalizada. Esta consiste en la identificación del álgebra elemental con el "simbolismo algebraico" (o lenguaje algebraico), en contraposición a, pero también como desarrollo de, un supuesto "lenguaje aritmético". En el mismo trabajo se destacan algunas de las características principales de esta interpretación del álgebra que recordamos concisamente a continuación:

a) El álgebra escolar se construye en un contexto exclusivamente numérico, a modo de generalización de los cálculos con números y de la traducción de expresiones numérico-verbales. Se la considera como un mero epifenómeno de la aritmética. 
b) Se considera, de manera simplista, que las expresiones algebraicas surgen ante la necesidad de representar y manipular números desconocidos, se supone que ésta es su razón de ser.

c) Las tareas específicamente "algebraicas" se reducen a la manipulación formal de expresiones algebraicas con letras y números (lo que se suele denominar "cálculo algebraico") y a la resolución de ecuaciones.

d) En la escritura y manipulación de expresiones algebraicas, la aritmética generalizada hace una distinción absoluta entre los datos conocidos (valores numéricos) por un lado y las incógnitas por otro.

e) Una ecuación se interpreta como una igualdad numérica que se cumple para algunos valores concretos de las incógnitas.

Encontramos un gran número de investigaciones en didáctica que asumen como propia (o por lo menos no cuestionan) esta visión del álgebra como aritmética generalizada y toman como objeto de estudio los obstáculos que surgen en el paso de la aritmética al álgebra. Es el caso, por ejemplo, de algunas perspectivas psicolingüístic as cuando estudian el paso del "lenguaje aritmético" al "lenguaje algebraico" así como la influencia del "lenguaje natural" en dicho tránsito, siempre enmarcado en una actividad conceptual (Clement, 1982), o bien cuando abordan el problema de la traducción al lenguaje algebraico de proposiciones numéricas enunciadas en lenguaje natural (Bell y Malone, 1993; Burton, 1988). También trabajos como los de Warren (2001, 2004), entre otros, postulan que muchas dificultades al pasar de la aritmética al álgebra son debidas a la falta de una base aritmética adecuada y a la desconexión entre los conocimientos aritméticos y algebraicos de los alumnos.

Para salvar las dificultades surgidas en el paso entre estas dos áreas de la matemática enseñada algunos investigadores proponen llevar a cabo una algebrización de la aritmética, es decir, introducir progresivamente (y de manera formal) en el cálculo con números la sintaxis propia del cálculo algebraico (Kaput, 2000). Estudios recientes en esta misma línea postulan que la adquisición en edad temprana del pensamiento algebraico evitaría muchas de las dificultades que muestran los alumnos en Secundaria. Dicha creencia ha dado fuerza a la propuesta de cambio curricular llamada Early Algebra. 
Este enfoque propone la introducción del pensamiento algebraico desde los primeros cursos escolares, de forma integrada con la actividad matemática relativa a las diferentes áreas de la matemática en esta etapa. En particular, autores como Carraher, Schliemann y Brizuela (2006), Kaput (2000), Malara (2003), Subramaniam (2004) y Warren (2004) han centrado su investigación en el pensamiento algebraico que puede ser promovido en el contexto numérico a partir de actividades que faciliten la transición entre la aritmética y el álgebra. Estos autores proponen adoptar un enfoque estructural para romper con el énfasis computacional que predomina en los primeros cursos escolares y favorecer el desarrollo de modos de pensamiento algebraico, ayudando así a los alumnos a familiarizarse con las estructuras que subyacen en las operaciones matemátic as y sus propiedades. Este enfoque consiste básicamente en proponer a los alumnos problemas en los que se deben extraer patrones numéricos y relaciones funcionales expresadas verbalmente. En una comparación superficial del enfoque Early Algebra con la interpretación del álgebra elemental que se desprende del MER propuesto, podemos afirmar que en ambos casos se postula cierta "algebrización de la aritmética", aunque el modelo epistemológico que se desprende del MER no comporta una relación privilegiada del álgebra elemental con la aritmética.

\section{Síntesis de Resultados}

Utilizando como sistema de referencia el MER en torno al álgebra elemental descrito en la sección 3, hemos caracterizado lo que, en la práctica, constituye la razón de ser del álgebra elemental en la enseñanza secundaria actual: el tipo de herramientas algebraic as que se proponen para ser enseñadas actualmente en la ESO no va mucho más allá del trabajo formal dentro de la segunda etapa del proceso de algebrización, limitándose además a la resolución de ecuaciones de primer y segundo grado, con una variable y sin parámetros. Además, la transición hacia el cálculo con funciones no acaba de estar motivada por las limitaciones del cálculo ecuacional. No es extraño pues que las manipulaciones algebraicas acaben siendo un mundo cerrado (Chevallard, 1989) con la única posibilidad de adquirir sentido por sí mismas y en sí mismas.

El examen de los procesos transpositivos que han afectado esta área curricular, y que hemos llevado a cabo en la sección 2 , ha mostrado la poca 
consciencia de la función que podría desempeñar el álgebra elemental en el desarrollo de la actividad matemática que los alumnos deben llevar a cabo. Surge aquí una difícil cuestión relativa a las posibles vías de evolución: ¿Cómo puede la investigación en didáctica contribuir a modificar esta situación en una dirección determinada? El tipo de análisis que propone la TAD y que hemos presentado parcialmente en este trabajo requiere en primer lugar una emancipación radical por parte de la investigación respecto de la visión sobre las matemáticas escolares que predomina en el sistema de enseñanza $\mathrm{y}$, parcialmente, en la matemática sabia. La construcción del MER a partir de datos provenientes de todas las etapas del proceso de transposición didáctica es un instrumento importante para posibilitar esta emancipación en relación a la ideología de las instituciones que forman parte de su objeto de estudio.

Ahora bien, el objetivo final de la investigación didáctica no es únicamente describir y explicar cómo son las cosas y por qué son así, sino mostrar también posibles vías de evolución. Resulta para ello fundamental el análisis ecológico que hemos descrito en la sección 4. Poco podremos cambiar si desconocemos los obstáculos o barreras de todo tipo (tanto material como ideológico o conceptual) que limitan las actividades que es posible llevar a cabo en el aula.

Dada la rigidez del sistema escolar, es previsible la aparición de múltiples dificultades ante cualquier intento de hacer evolucionar el álgebra escolar hacia formas más funcionales (en contraposición de las actividades algebraicas mayoritariamente formales). Nuestro análisis sugiere que para que fuese posible integrar el álgebra como instrumento de modelización en la ESO se requeriría un cambio cultural que provocase cambios significativos de los modelos epistemológicos y didácticos dominantes actualmente en las instituciones escolares lo que, obviamente, plantea enormes dificultades.

\section{Notas}

${ }^{1}$ La delimitación de las etapas del modelo propuesto es relativamente arbitraria ya que, como cualquier modelo, responde a una intención de abordar cierto fenómeno. El lector encontrará una descripción más detallada en Ruiz-Munzón (2010). 
126 Ruiz Munzón et al - El Problema Didáctico del Álgebra Elemental

\section{Referencias}

Artigue, M., Assude, T., Grugeon, B. y Lenfant, A. (2001). Teaching and learning algebra: Approaching complexity through complementary perspectives. En H. Chick, K. Stacey, J. Vincent, J. Vincent (Eds.), The future of the teaching and learning of algebra Proceedings of the $12^{\text {th }}$ ICMI Study Conference (vol.1, pp. 21-32). Melbourne, Australia: The University of Melbourne.

Assude, T., Coppe, S. y Pressiat, A. (2012). Tendances de l'enseignement de l'algèbre élémentaire au Collège: Atomisation et réduction. En L. Coulange, J.-P. Drouhard, J.-L. Dorier, A. Robert (Eds) Recherche en didactique des mathématiques. Enseignement de l'algèbre, élémentaire. Bilan et perspectives (pp. 41-62). Grenoble: La Pensée Sauvage.

Atiyah, M. (2002). Las matemáticas en el siglo XX. Traducción en Números. Revista de didáctica de las matemáticas, 50, 35-55. Recuperado de http://bit.ly/1JLSth8

Behr, M., Erlwanger, S. y Nichols, E. (1980). How children view the equal sign. Mathematics Teaching, 92, 13-15.

Bell, A. y Malone, J. (1993). Learning the language of algebra, Proceedings of PME XVII (vol. I, pp. 130-137). Tsukuba, Japón.

Bednarz, N. (2001). A problem-solving approach to algebra. Accounting for the reasonings and notations developed by students. En H. Chick, K. Stacey, J. Vincent y J. Vincent (Eds.), The future of the teaching and learning of algebra Proceedings of the $12^{\text {th }}$ ICMI Study Conference (vol. 1, pp. 69-78). Melbourne, Australia: The University of Melbourne.

Blanco, L. y Garrote, M. (2007). Difficulties in Learning Inequalities in Students of the First Year of Pre-University Education in Spain. Eurasia Journal of Mathematics, Science y Technology Education, 3(3), 221-229. Recuperado de http://www.ejmste.com/com33.aspx

Bolea, P. (2003). El proceso de algebrización de organizaciones matemáticas escolares. Monografía del Seminario Matemático García de Galdeano, 29. Departamento de Matemáticas. Universidad de Zaragoza.

Bolea, P., Bosch, M. y Gascón, J. (1998). Le caractère problématique du processus d'algébrisation. Proporcionnalité et grandeurs dans 
l'enseignement obligatoire, Actes de la IXème école d'été de didactique des mathématiques, ARDM, 153-159.

Bolea, P., Bosch, M. y Gascón, J. (2001a). La transposición didáctica de organizaciones matemáticas en proceso de algebrización. Recherches en Didactique des Mathématiques, 21(3), 247-304. Recuperado de http://rdm.penseesauvage.com/-Volume-21-.html

Bolea, P., Bosch, M. y Gascón, J. (2001b). ¿Cómo se construyen los problemas en Didáctica de las Matemátic as? Educación Matemática, 13(3), 22-63. Recuperado de http://w ww.revista-educacionmatematica.com/volumenes/volumen-13/numero-3

Booth, L. (1984). Algebra. Children's Strategies and Errors. Windsor, NFER-Nelson.

Bosch, M. (1994). La dimensión ostensiva en la actividad matemática. El caso de la proporcionalidad. Tesis doctoral no publicada. Universitat Autònoma de Barcelona.

Burton, M. (1988). A linguistic basis for the student difficulties with algebra. For the Learning of Mathematics, 8, 2-7. Recuperado de http://flmjournal.org/index.php?do=show \&lang=en \&showMenu=8\%2C1

Carraher, D. W., Schliemann, A. D., Brizuela, B. M. y Earnest, D. (2006). Aritmetic an Algebra in Early Mathematics Education. Journal for Research in Mathematics Education, 37(2), 87-115. doi: $10.2307 / 30034843$

Chevallard, Y. (1984). Le passage de l'arithmétique a l'algébrique dans l'enseignement des mathématiques au collège - Première partie. L'évolution de la transposition didactique. Petit x, 5, 51-94. Recuperado de http://www-irem.ujfgrenoble.fr/revues/revue_x/fic/5/5x3.pdf

Chevallard, Y. (1985). La Transposition Didactique. Du savoir savant au savoir enseigné. Grenoble, France: La Pensée Sauvage ( $2^{a}$ edición 1992).

Chevallard, Y. (1989). Le passage de l'arithmétique a l'algébrique dans l'enseignement des mathématiques au collège - Deuxième partie. Perspectives curriculaires: la notion de modélisation. Petit x, 19, 4575. Recuperado de http://w w w-irem.ujfgrenoble.fr/revues/revue_x/fic/19/19x5.pdf 
Chevallard, Y. (1990). Le passage de l'arithmétique a l'algébrique dans l'enseignement des mathématiques au collège - Troisième partie.

Perspectives curriculaires: vois d'attaque et problèmes didactiques.

Petit $x, 23,5-38$. Recuperado de http://www-irem.ujf-

grenoble.fr/revues/revue_x/fic/19/19x5.pdf

Chevallard, Y. (2002). Organiser l'étude. 3. Écologie regulation. In J.-L.

Dorier, Artaud, M., Artigue, M., Berthelot, R. Floris, R. (Eds) Actes de la 1le École d'Été de didactique des mathématiques. Grenoble, France: La Pensée sauvage (pp. 41-56).

Chevallard, Y. (2007). Passé et present de la Théorie Anthropologique de Didactique. En A. Estepa, L. Ruiz, F. J. García (Eds.), Sociedad, escuela y matemáticas. Aportaciones de la Teoría Antropológica de lo Didáctico (TAD) (pp. 705-746). Jaén: Publicaciones de la Universidad de Jaén.

Chevallard, Y. y Bosch, M. (2012). L'algèbre entre effacement et réaffirmation aspects critiques de l'offre scolaire d'algèbre. En $\mathrm{L}$. Coulange, J.-P. Drouhard, J.-L. Dorier, A. Robert (Eds.) Recherches en Didactique des Mathématiques. Enseignement de l'algèbre élémentaire: bilan et perspectives (pp. 19-39). Tesis doctoral no publicada. Université Joseph-Fourier-Grenoble I.

Clement, J. (1982). Algebra word problem solutions: Thought processes underlying a common misconception. Journal for Research in Mathematics Education, 13, 16-30. doi: 10.2307/748434

Coulange, L., Drouhard, J.-P., Dorier, J.-L. y Robert, A. (Eds.) (2012). Recherches en Didactique des Mathématiques, Numéro spécial horssérie, Enseignement de l'algèbre élémentaire: bilan et perspectives. Grenoble: La Pensée Sauvage.

Derrida, J. (1967). De la grammatologie. París: Les Editions de Minuit. Filloy, E. y Rojano, T. (1989). Solving equations: the transition from arithmetic to algebra. For the Learning of Mathematics, 9, 19-25. Recuperado de http://flmjournal.org/index.php?do=show \&lang=en\&show Menu=9\%2C 2

Filloy, E., Rojano, T. y Puig, L. (2008). Educational Algebra. A Theoretical and Empirical Approach. New York: Springer.

Gascón, J. (1993). Desarrollo del conocimiento matemático y análisis didáctico: Del patrón análisis-síntesis a la génesis del lenguaje algebraico. Recherches en didactique des mathématiques, 13(3), 295- 
332. Recuperado de http://rdm.penseesauvage.com/-Volume-13.html

Gascón, J. (1994-95). Un nouveau modèle de l'algèbre élémentaire comme alternative à l'《 arithmétique généralisée ». Petit $x, 37,43-63$.

Recuperado de http://www-irem.ujf-

grenoble.fr/revues/revue_x/fic/37/37x4.pdf

Gascón, J. (1999). La naturaleza prealgebraica de la matemática escolar.

Educación matemática, 11(1), 77-88. Recuperado de http://ww w .revista-educacion-matematica.com/volumenes/volumen11/numero-1

Gascón, J. (2011). Las tres dimensiones fundamentales de un problema didáctico. El caso del álgebra elemental. Revista Latinoamericana de Investigación en Matemática Educativa, RELIME, 14 (2), 203-231.

Recuperado de http://www.clame.org.mx/relime.htm

González Astudillo, M. T. (2006). La matemática moderna en España.

Unión. Revista Iberoamericanda de educación matemática, 6, 63-71. Recuperado de http://ww w. fisem.org/www/union/revista6.php

Grugeon, B., Pilet, J., Chenevotot, F. y Delozanne, E. (2012). Diagnostic et parcours différenciés d'enseignement en algèbre élémentaire. En L. Coulange, J.-P. Drouhard, J.-L. Dorier, A. Robert (Eds.) Recherches en Didactique des Mathématiques. Enseignement de l'algèbre élémentaire: bilan et perspectives (pp. 137-162). Grenoble: La Pensée Sauvage.

Kaput, J. (2000). Transforming algebra from an engine of inequality to an engine of mathematical power by "algebrafying" the K-12 curriculum. Dartmouth, MA: National Center for Improving Student Learning and Achievement in Mathematics and Science.

Kieran, C. (1981). Concepts associated with the equality symbol. Educational Studies in Mathematics, 12(3), 317-326. doi: 10.1007/BF00311062

Lins, R. y Kaput, J. (2004). The early development of algebraic reasoning: the current state of the field. En K. Stacey, H. Chick y M. Kendal (Eds), The teaching and learning of algebra. The 12th ICMI Study (pp.47-70). Norwell, MA: Kluwer Academic Publishers.

Malara, N.A. (2003). Dialectics between theory and practice: theoretical issues and aspects of practice from an Early Algebra Project. En N. Pateman, G. Dougherty y J. Zilliox (Eds.), Proceedings of the $27^{\text {th }}$ 
Conference of the International Group for the Psychology of Mathematics Education North America (vol. 1, pp. 33-48).

Malisani, E. y Spagnolo, F. (2009). From arithmetical thought to algebraic thought: The role of the "variable". Educational Studies in Mathematics, 71, 19-41. doi: 10.1007/s10649-008-9157-x

Puig, L. (2012). Observaciones acerca del propósito del álgebra educativa. In A. Estepa, Á. Contreras, J. Deulofeu y L. Ordóñez (Eds.), Investigación en Educación Matemática XVI (pp. 1-20). Jaén: SEIEM.

Ruiz-Munzón, N. (2010). La introducción elemental y su desarrollo hacia la modelización funcional. Tesis Doctoral no publicada. Universitat Autònoma de Barcelona.

Ruiz-Munzon, N., Matheron, Y., Bosch, M. y Gascón, J. (2012). Autour de l'algèbre : les entiers relatifs et la modelisation algébricofonctionnelle. In L. Coulange, J.-P. Drouhard, J.-L. Dorier, A. Robert (Eds.) Recherches en Didactique des Mathématiques. Enseignement de l'algèbre élémentaire: bilan et perspectives. Grenoble: La Pensée Sauvage.

Sesa, C. (2005). Iniciación al estudio didáctico del Álgebra, Buenos Aires: Libros del Zorzal.

Sfard, A. y Linchevski, L. (1994). The gains and the pitfalls of reification. The case of algebra. Educational Studies in Mathematics, 26, 191228. doi: 10.1007/978-94-017-2057-1_4

Subramaniam, K. (2004). Naming practices that support reasoning about and with expressions. $10^{\text {th }}$ International Congress on Mathematical Education. Copenhague.

Trigueros, M., Reyes, A., Ursini, S. y Quintero, R. (1996). Diseño de un cuestionario de diagnóstico acerca del manejo del concepto de variable en el álgebra. Enseñanza de las ciencias, 14(3), 351-363. Recuperado de http://ensciencias.uab.es

Vergnaud, G. (1985). Understanding mathematics at the secondary-school level. En A. Bell, B. Low y J. Kilpatrick (comps.), Theory Research y Practice in Mathematical Education, (pp.27-45). University of Nottingham, Shell Center for Mathematical Education.

Warren, E. (2001). Algebraic understanding and the importance of operation sense. En M. Heuvel-Penhuizen (Ed.), Proceedings of the 
25th International Group for the Psychology of Mathematics Education (vol. 4, pp. 399-406). Utrecht. Freudenthal Institute. Warren, E. (2004). Generalizing arithmetic: supporting the process in the early years. En M. Johnsen y A. Berit (Eds.), Proceedings of the $28^{\text {th }}$ International Group for the Psychology of Mathematics Education, Vol. 4, 417-424. Bergen (Noruega): Bergen University College.

Noemí Ruiz Munzón es profesora asociada en la Escola Superior de Ciències Socials i de l'Empresa-Tecnocampus, de la Universitat Pompeu Fabra, España.

Marianna Bosch es catedrática de Métodos Cuantitativos, en el IQS School of Management, de la Universitat Ramon Llull, España.

Josep Gascón es professor agregado laboral, en el Departamento de Matemáticas, de la Universitat Autònoma de Barcelona, España.

Contact Address: La correspondencia directa sobre este artículo, se debe enviar al autor/a. Dirección postal: Av. Ernest Lluch, 32, 08032 Barcelona (España). Email: nruiz@ tecnocampus.cat 